\title{
Understanding the psychiatric symptoms of COVID-19: a meta-analysis of studies assessing psychiatric symptoms in Chinese patients with and survivors of COVID-19 and SARS by using the Symptom Checklist-90-Revised
}

\author{
Qin Xie ${ }^{1,2}$, Xiao-Bo Liu' ${ }^{1,2}$, Yan-Min X $\mathrm{u}^{1,2}$ and Bao-Liang Zhong $\mathbb{B}^{1,2}$
}

\begin{abstract}
Understanding the psychiatric symptoms of COVID-19 could facilitate the clinical management of COVID-19 patients. However, the profile of psychiatric symptoms among COVID-19 patients has been understudied. We performed a meta-analysis of studies assessing psychiatric symptoms of COVID-19 and SARS patients and survivors by using the Symptom Checklist-90-Revised (SCL-90-R), an instrument covering a wide spectrum of psychiatric symptoms. Studies reporting SCL-90-R subscale scores among patients with and survivors of COVID-19 and SARS were retrieved from major English and Chinese literature databases. Patients' pooled SCL-90-R subscale scores were compared to the Chinese normative SCL-90-R data, and Cohen's $d$ values were calculated to indicate the severity of psychiatric symptoms. The Joanna Briggs Institute Critical Appraisal Checklist for Studies Reporting Prevalence Data was used to assess the quality of the included studies. The search yielded 25 Chinese studies with 1675 acute COVID-19 and 964 acute SARS patients, 30 COVID-19 and 552 SARS survivors during very early recovery (up to 1 month since discharge), 291 SARS survivors during early recovery (1-6 months after discharge), and 48 SARS survivors during late recovery (12 months after discharge). None of the included studies were rated as good quality. The ten SCL-90-R-defined psychiatric symptoms, which were of medium-to-severe severity $(d=0.68-3.01)$, were all exhibited in acute COVID-19 patients, and the severity of these symptoms decreased to mild-to-medium during very early recovery $(d=0.17-0.73)$. SARS patients presented eight psychiatric symptoms with mild-to-severe severity during the acute stage $(d=0.43-1.88)$, and thereafter, the severity of symptoms decreased over the follow-up period. However, somatization $(d=0.30)$ and anxiety $(d=0.28)$ remained at mild levels during late recovery. A wide variety of severe psychiatric symptoms have been reported by acute COVID-19 patients, and these symptoms, despite decreasing in severity, persist in very early recovery. The changing trajectory observed with SARS suggests that psychiatric symptoms of COVID-19 may persist for a long time after discharge, and therefore, periodic monitoring of psychiatric symptoms, psychosocial support, and psychiatric treatment (when necessary) may be necessary for COVID-19 patients from the acute to convalescent stages.
\end{abstract}

Correspondence: Bao-Liang Zhong (haizhilan@gmail.com)

'Department of Psychiatry, Wuhan Mental Health Center, Wuhan, Hubei province, China

${ }^{2}$ Department of Psychiatry, Affiliated Wuhan Mental Health Center, Tongji Medical College of Huazhong University of Science \& Technology, Wuhan, Hubei Province, China

These authors contributed equally: Qin Xie, Xiao-Bo Liu

\section{Introduction}

Psychiatric presentations and mental disorders are common among COVID-19 patients ${ }^{1,2}$. Empirical data have shown that $43.1 \%$ and $40.2 \%$ of COVID-19 patients suffer from depressive symptoms and mental illnesses,

\section{(-) The Author(s) 2021}

(c) (i) Open Access This article is licensed under a Creative Commons Attribution 4.0 International License, which permits use, sharing, adaptation, distribution and reproduction cc) in any medium or format, as long as you give appropriate credit to the original author(s) and the source, provide a link to the Creative Commons license, and indicate if changes were made. The images or other third party material in this article are included in the article's Creative Commons license, unless indicated otherwise in a credit line to the material. If material is not included in the article's Creative Commons license and your intended use is not permitted by statutory regulation or exceeds the permitted use, you will need to obtain permission directly from the copyright holder. To view a copy of this license, visit http://creativecommons.org/licenses/by/4.0/. 
respectively ${ }^{3,4}$. Cooccurring mental health problems complicate the respiratory management of COVID-19 patients and negatively affect the prognosis of COVID-19 (refs. ${ }^{5,6}$ ). For example, patients who develop psychotic symptoms may not adhere to respiratory treatment or may even threaten the safety of frontline medical staff, and depressed survivors may not be able to return to work. Therefore, a timely and detailed assessment of mental health morbidities is important for the effective clinical management of COVID-19 patients and survivors.

Available research on mental health problems associated with COVID-19 is limited to case reports/series, self-report questionnaire surveys, and mental disorder surveys ${ }^{3,4,7-10}$. Because case reports/series are subject to selection bias, i.e., reporting unusual cases with manic episodes, the generalizability of their findings is poor. Most prior questionnaire surveys focused on depressive and anxiety symptoms, so data regarding psychiatric symptoms other than depression and anxiety associated with COVID-19 are still limited. Despite having first-hand data on a variety of mental disorders, mental disorder surveys have provided little information on subclinical psychiatric symptoms of COVID-19. According to the mental health continuum model, psychiatric symptoms are early signs of mental disorders, and persons with long-lasting and severe symptoms are more likely to develop mental disorders ${ }^{11}$. Therefore, the assessment and identification of psychiatric symptoms have clinical implications for early psychological interventions for COVID-19 patients. To the best of our knowledge, only one study has assessed psychiatric symptoms of COVID-19 patients by using psychiatric interviews, and 11 symptoms were identified in this patient population, including insomnia, aggressive behaviors, delusions, and hallucinations ${ }^{5}$. However, because the sample size of this study was small $(n=25)$ and its participants were COVID-19 patients who received psychiatric inpatient care for comorbid firstonset mental disorders, its findings are difficult to generalize to general COVID-19 patients. Therefore, data regarding the full spectrum of psychiatric symptoms among persons with COVID-19 are still very limited.

As of March 25, 2021, the total number of globally confirmed cases of COVID-19 had been 125,436,393, of whom 10,130,4931 survived, making postdischarge rehabilitation services an urgent clinical priority ${ }^{12-14}$. Unfortunately, the long-term mental health sequelae of COVID-19 are still poorly understood due to the lack of empirical studies ${ }^{15,16}$.

The Symptom Checklist-90-Revised (SCL-90-R) is a widely used self-report scale to assess a broad range of psychological problems and symptoms of psychopathology $^{17}$. The SCL-90-R has been used to assess the psychiatric symptoms of both clinical and nonclinical populations, including SARS patients ${ }^{18}$. There have been some studies investigating psychiatric symptoms of
COVID-19 by using the SCL-90- $\mathrm{R}^{19}$. This provided us with an opportunity to examine the profile of psychiatric symptoms of COVID-19; however, these studies were limited by their small sample sizes and wide variations in SCL-90-R test results ${ }^{20-22}$. For example, Xing and colleagues assessed the psychiatric symptoms of 42 acute COVID-19 patients and reported that the mean score of the depression subscale of the SCL-90-R was 1.36, while Wang and colleagues investigated 40 acute COVID-19 patients and found the corresponding mean score was 3.15 , which was an over twofold difference ${ }^{21,23}$.

To fill the abovementioned knowledge gap, we performed a meta-analysis of clinical studies assessing psychiatric symptoms in COVID-19 patients, as denoted by the SCL-90-R scores. Given that COVID-19 and SARS have similar pathophysiological mechanisms, we also quantitatively combined the SCL-90-R test results of SARS patients, in particular SARS survivors, which may inform the planning of follow-up services for COVID-19 survivors.

\section{Methods \\ Inclusion and exclusion criteria}

The inclusion criteria were as follows: (a) studies were cross-sectional studies or baseline assessments of cohort or interventional studies published in English or Chinese; (b) study participants were adults with a current or past diagnosis of COVID-19 or SARS; (c) studies included a minimum sample of ten patients; (d) participants' psychiatric symptoms were assessed with the SCL-90-R; and (e) studies presented the mean scores [with standard deviations (SDs)] of at least one of the ten primary subscales of the SCL-90-R: somatization, obsessive-compulsive, interpersonal sensitivity, depression, anxiety, hostility, phobic anxiety, paranoid ideation, psychoticism, and appetite and sleep. We excluded duplicate reports and studies using samples of COVID-19 patients referred for psychiatric consultation.

\section{Literature search}

Major Chinese and English databases were searched from their inception to March 1, 2021. These databases were China National Knowledge Infrastructure, Wanfang Data Knowledge Service Platform, CQVIP, SinoMed, PubMed, EMBASE, and PsycInfo. The following search terms were used: "COVID-19" or "2019-nCoV" or "coronavirus" or "SARS" or "severe acute respiratory syndrome" and "SCL-90" or "Symptom Checklist". Reference lists of the retrieved papers were also hand-searched to avoid missing certain records.

\section{Data extraction}

Variables collected from each included study were first author, publication year, coronavirus disease (COVID-19 
vs. SARS), type of participants (patients vs. survivors), sample size, numbers of male and female participants, and SCL-90-R subscale scores (mean $\pm \mathrm{SD}$ ). In accordance with Gardner and Moallef ${ }^{24}$, the stage of coronavirus disease was roughly divided into the acute stage (i.e., at admission and during hospital stay), very early recovery stage (at discharge and up to 1 month after discharge), early recovery stage (1-6 months postdischarge), and late recovery stage (6-12 months postdischarge).

\section{Quality assessment}

The Joanna Briggs Institute Critical Appraisal Checklist for Studies Reporting Prevalence Data (abbreviated as "JBI checklist") was used to assess the quality of the included studies $^{25}$. The JBI checklist has nine methodological items, with each having four answer choices (yes, no, unclear, or not applicable): sample frame, sampling, sample size, description of subjects and setting, sample coverage of the data analysis, validity of the method for assessing the outcome, standardization and reliability of the method for assessing outcome, statistical analysis, and response rate. The quality score is the total number of "yes" answers, which can range from 0 to 9 , with higher scores suggesting higher quality ${ }^{26}$. A JBI score of "9" suggests "good quality".

The first and second authors independently performed the literature search, selected eligible studies, extracted data, and assessed the quality of the included studies. Any discrepancies were resolved by their consensus.

\section{Statistical analysis}

We used the "metamean" package of $\mathrm{R}$ 4.0.2 to produce pooled estimates and their 95\% confidence intervals (CIs) for the SCL-90-R subscale scores, according to coronavirus disease (COVID-19 vs. SARS) and stage of coronavirus disease (acute vs. recovery). When there was little evidence of heterogeneity $\left(I^{2} \leq 50 \%\right)$, a fixed effects model was used to produce the combined estimates; otherwise, a random effects model was used. Publication bias was tested by using Egger's test when the total number of included studies was ten or more for the metaanalysis of a SCL-90-R subscale; otherwise, publication bias was not tested. Due to the small number of included studies, sources of heterogeneity in the meta-analyses were not explored. To gauge magnitudes of differences in symptom severity between patients and the general population, Cohen's $d$ values were calculated for SCL-90$\mathrm{R}$ subscale scores, with $0.20-0.49,0.50-0.79$, and $0.80+$ being operationally defined as mild, medium, and severe symptoms, respectively ${ }^{27}$. We introduced two Chinese SCL-90-R normative data in 2006 and 2014, as the comparison references for the SCL-90-R scores of SARS patients and survivors, and COVID-19 patients and survivors, respectively ${ }^{28,29}$.

\section{Results}

The process of study identification and inclusion is shown in Fig. 1. Finally, 25 studies were eligible for this study, including 12 studies with 1675 acute patients with COVID-19, 2 studies with 30 COVID-19 survivors during very early recovery, 7 studies with 964 acute patients with SARS, 7 studies with 552 SARS survivors during very early recovery, 4 studies with 291 SARS survivors during early recovery, and 1 study with 48 SARS survivors during late recovery ${ }^{20-23,30-50}$. Participants in the included studies were all Chinese patients or survivors. The quality scores of the included studies ranged from 2 to 7 . Detailed characteristics and quality assessment of the included studies are displayed in Table 1.

Acute COVID-19 patients presented all the ten SCL-90R-defined psychiatric symptoms. Nearly all the psychiatric symptoms of COVID-19 were severe during the acute stage, and their severity decreased to mild-to-medium during very early recovery: somatization $(d=2.33$ and 0.55 , respectively), obsessive-compulsive $(d=0.98$ and $0.17)$, interpersonal sensitivity $(d=1.28$ and 0.43$)$, depression $(d=1.56$ and 0.44$)$, anxiety $(d=2.27$ and $0.64)$, hostility $(d=0.97$ and 0.36$)$, phobia $(d=3.01$ and $0.73)$, paranoid ideation ( $d=0.68$ and 0.45$)$, psychoticism $(d=0.83$ and 0.47$)$, and appetite and sleep $(d=1.74$ and 0.19; Table 2 and Fig. 2).

With the exception of paranoid ideation $(d=0.04$ and 0.07 ), the remaining eight psychiatric symptoms in acute SARS patients were medium-to-severe and symptom severity during very early recovery, while lower, had remained at medium-to-severe levels: somatization $(d=$ 1.65 and 1.01), obsessive-compulsive ( $d=0.43$ and 0.33$)$, interpersonal sensitivity $(d=0.55$ and 0.41$)$, depression $(d=1.44$ and 0.74$)$, anxiety ( $d=1.88$ and 1.27$)$, hostility $(d=0.43$ and 0.32$)$, phobia $(d=1.15$ and 0.53$)$, and psychoticism $(d=0.56$ and 0.53$)$. Despite subsequent reductions in symptom severity in SARS, anxiety remained to be severe during early recovery $(d=0.82)$; somatization, obsessive-compulsive, depression, phobia, and paranoid ideation decreased to mild-to-medium during early recovery $(d=0.22-0.57)$; and somatization $(d=0.30)$ and anxiety $(d=0.28)$ remained at mild levels during late recovery (Table 2 and Fig. 3).

No significant publication bias was detected in metaanalyses of the SCL-90-R subscales of somatization, depression, anxiety, and hostility in acute patients with COVID-19 ( $P=0.208-0.856$; Table 2$)$.

\section{Discussion}

This study systematically summarized the profile of psychiatric symptoms in patients with COVID-19 and SARS, during both the acute and convalescent stages. We found that all ten SCL-90-R-defined psychiatric symptoms were exhibited in acute COVID-19 patients, and 


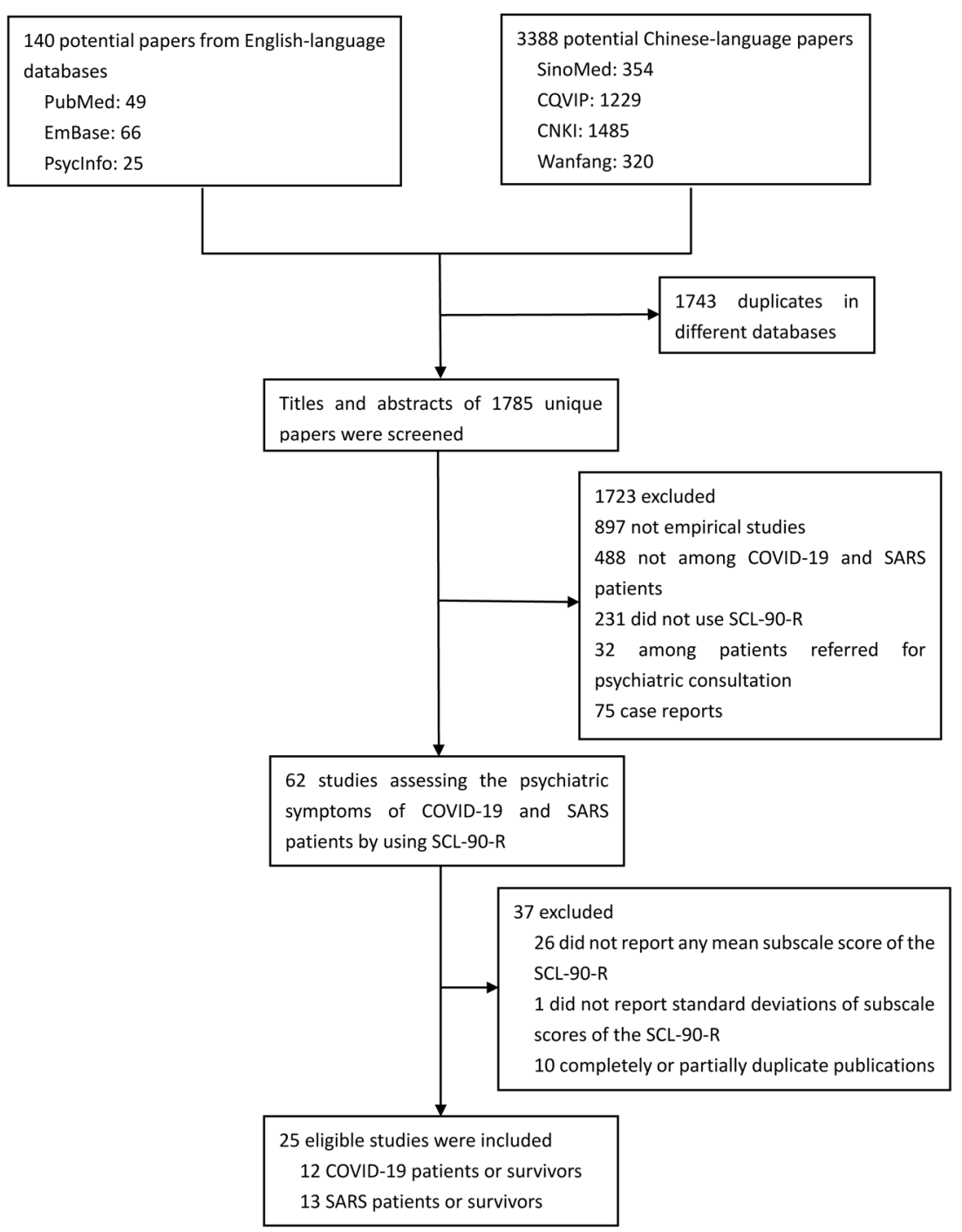

Fig. 1 Study identification and inclusion. Flowchart depicting the process of searching and screening for eligible studies of this meta-analysis.

nearly all these symptoms were severe. Acute SARS patients, while they had less severe psychiatric symptoms than COVID-19 patients, still presented many mediumto-severe symptoms. Thereafter, there was a trend toward overall declines in severity of psychiatric symptoms observed in survivors of both COVID-19 and SARS. However, most psychiatric symptoms of COVID-19 (i.e., phobia, anxiety, and somatization) were still mild-tomedium during very early recovery, and some symptoms of SARS, such as somatization, interpersonal sensitivity, and anxiety, still remained mild during late recovery.

The underlying physiological and psychosocial mechanisms associated with human coronavirus diseases could explain the high psychiatric symptom burden in acute patients with COVID-19 and SARS. For example, researchers have found positive SARS-CoV-2 RNA and parainfectious/postinfectious inflammatory changes in the cerebrospinal fluid of COVID-19 patients $^{51,52}$, so it is possible that psychiatric symptoms are part of the neuropsychiatric complications due to the central nervous system impact of viral infection ${ }^{53}$. Second, suffering from coronavirus diseases per se, a potentially life-threatening illness, is a stressful event for patients. Due to this, fear of death, worry about the infection of family members, despair, anger, frustration, and insomnia are common stress reactions in this patient population ${ }^{5}$. Third, physical discomfort and pain caused by COVID-19 and SARS could further exacerbate emotional reactions to coronavirus diseases. Fourth, because of the isolation treatment for patients, separation from family members and friends would increase the risk of feeling lonely and other mental health problems ${ }^{54}$. Fifth, antiviral treatment may also contribute to the psychiatric manifestations of patients; for example, there is evidence that both 


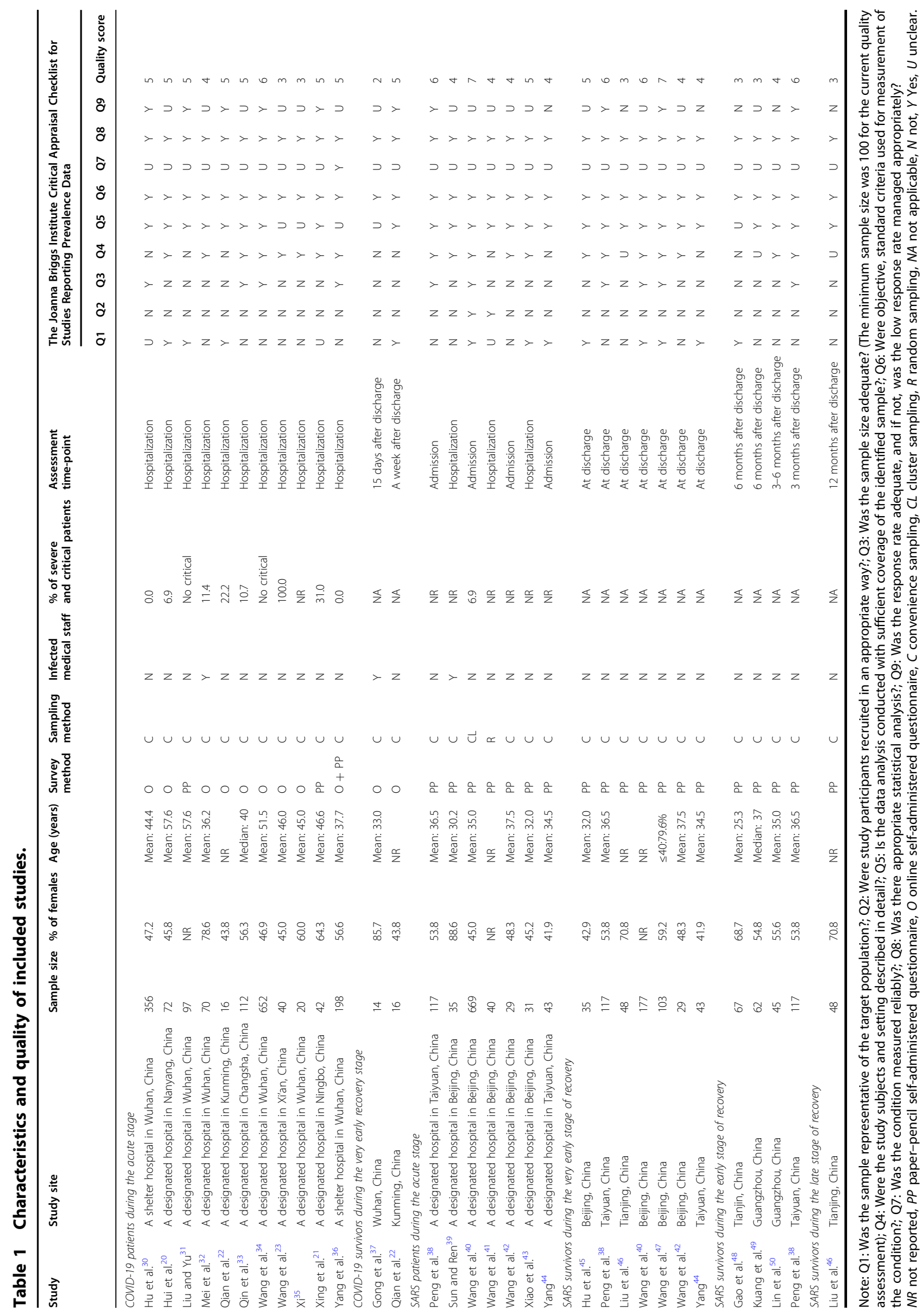




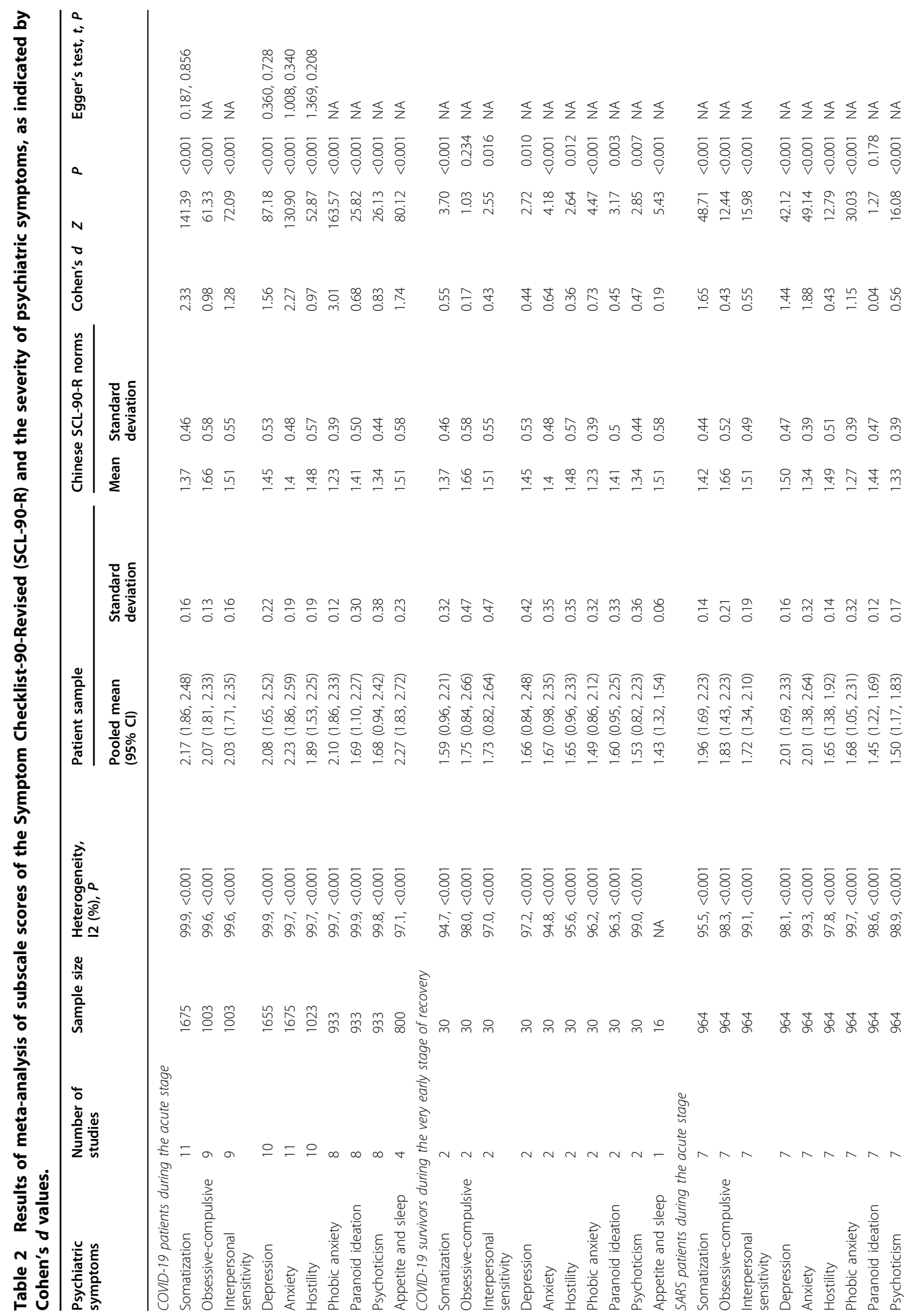




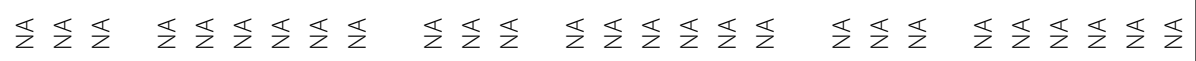

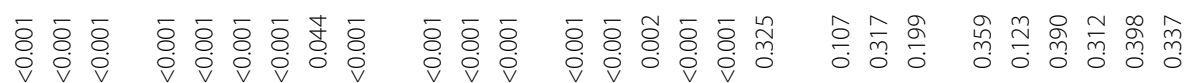

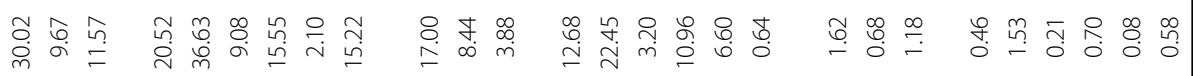

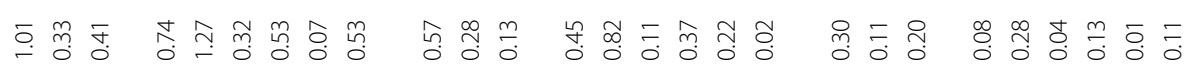

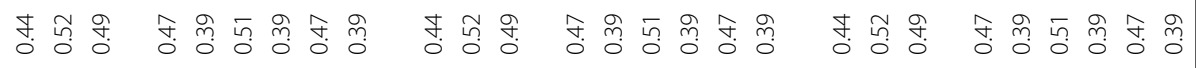

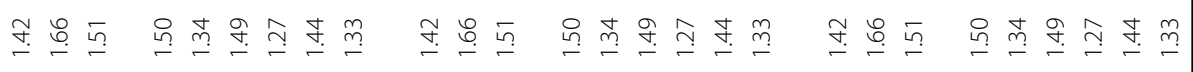

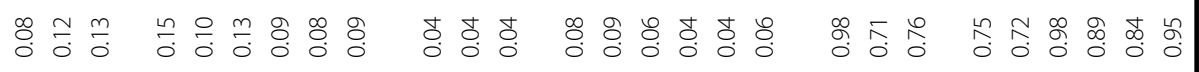

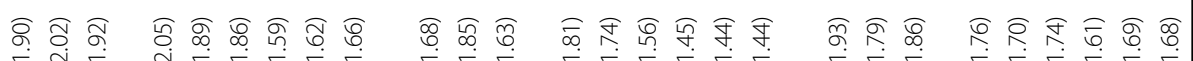

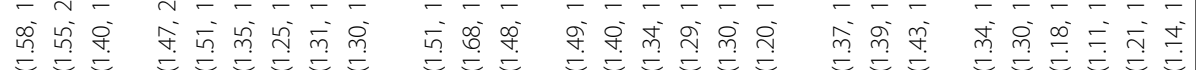

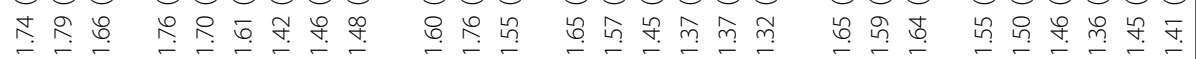

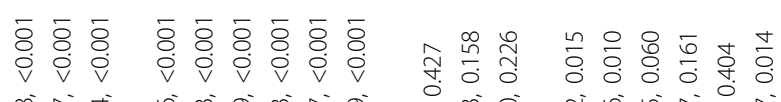

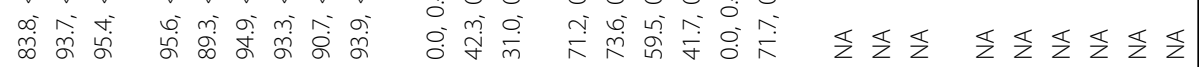

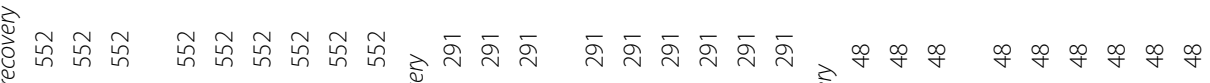

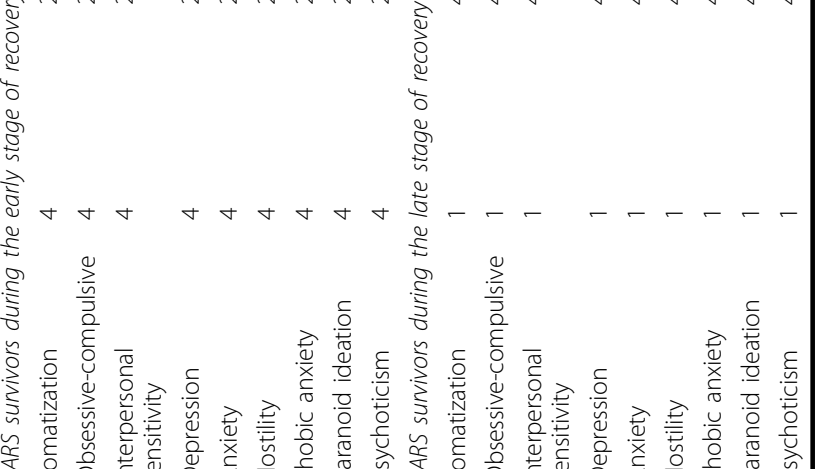



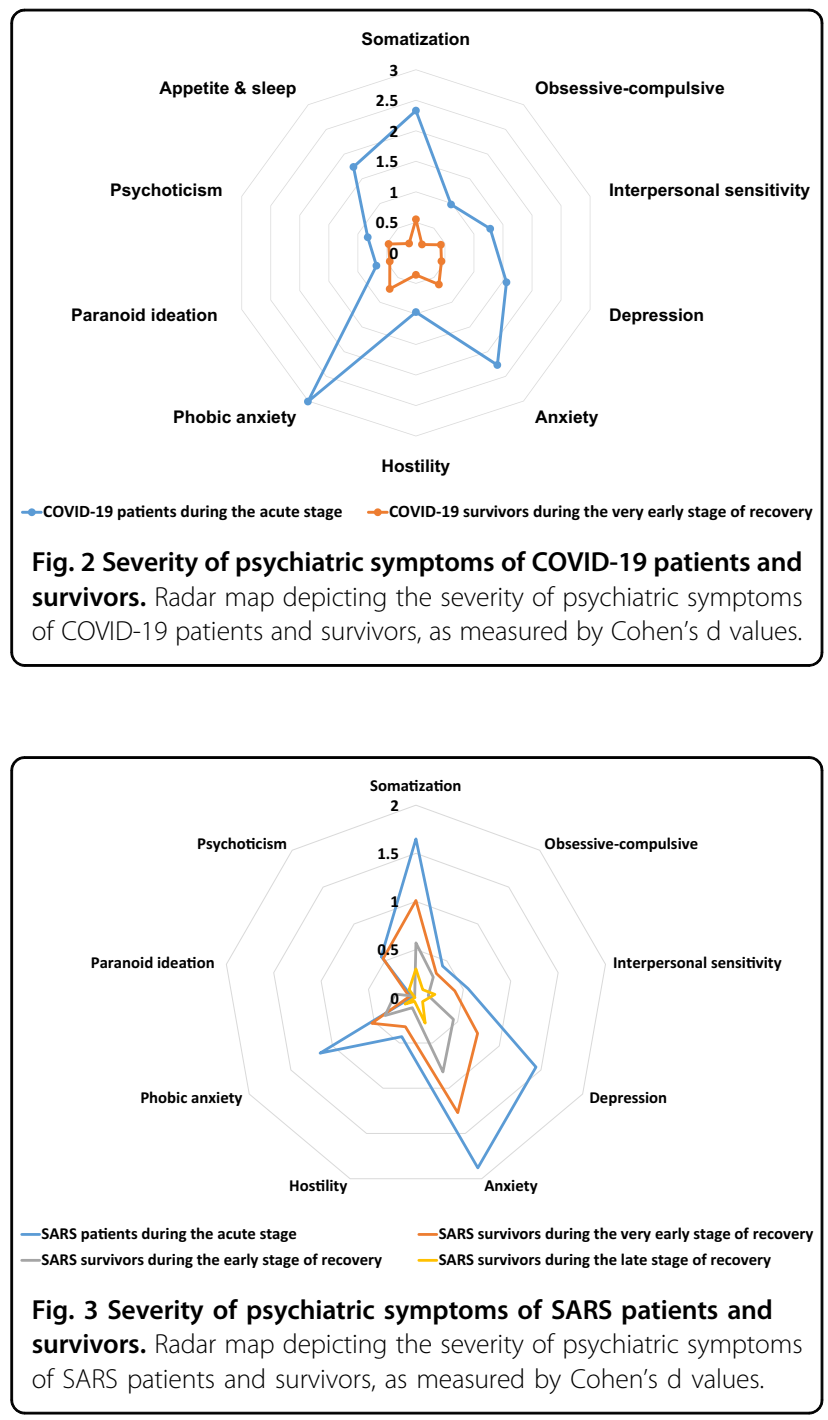

chloroquine and steroids could induce psychotic epi$\operatorname{sodes}^{55,56}$. We speculate that various severe psychiatric symptoms of COVID-19 are more likely to be the result of the joint effects of the abovementioned factors.

The relatively lower severity of psychiatric symptoms in SARS than in COVID-19 patients might be ascribed to the different psychosocial impacts of the two coronavirus diseases. For example, the SARS epidemic mainly affected people of Asian countries, but the COVID-19 pandemic has been a global crisis, affecting people of nearly all countries in the world. Compared to SARS, COVID-19 has quicker and wider transmission, disproportionate effects on older adults, and a high case-fatality rate in older adults ${ }^{57}$, and therefore, COVID-19 patients may have higher levels of psychological distress and fears of death than SARS patients. This may also explain why phobia was the most severe psychiatric symptom in
COVID-19 patients. Because of physical complications and discomfort caused by coronavirus diseases, severe symptoms of somatization with acute COVID-19 and SARS are expected. In addition, unlike the SARS epidemic in 2003, the ongoing COVID-19 pandemic is occurring concurrently with an "infodemic", where misinformation and disinformation can be easily and quickly disseminated via social media platforms ${ }^{58}$, which may further exacerbate the poor mental health of COVID-19 patients.

The reduced severity of psychiatric symptoms in COVID-19 and SARS patients from the acute stage to the late recovery stage suggests that psychiatric symptoms in the acute stage are mainly acute stress reactions and are therefore transient. These results indicate the importance of early mental health and psychosocial services at the stage of inpatient treatment. Nevertheless, the persistence of several psychiatric symptoms in SARS survivors throughout recovery might suggest the necessity of additional psychiatric symptom assessment and mental health services for the rehabilitation of COVID-19 survivors. Some postdischarge psychosocial factors may increase the risk of depression and other mental health problems in SARS survivors; for example, stigma associated with SARS and financial loss or even unemployment due to the past history of SARS infection. A recently published prognosis study reported that COVID-19 survivors were still suffering from fatigue, muscle weakness, sleep difficulties, depression, and anxiety 6 months after acute infection ${ }^{16}$. These findings are consistent with the residual psychiatric symptoms in recovered COVID-19 and SARS survivors in our study, such as somatization and anxiety.

This study has some limitations. First, the quality assessment results of the included studies suggest that, to a certain extent, these included studies are at risk of bias, so we must be cautious in generalizing the study findings. Second, owing to the lack of SCL-90-R data from COVID19 survivors during late recovery, and the very limited SCL-90-R data from SARS survivors during late recovery, more studies are warranted to investigate psychiatric symptoms of COVID-19 survivors, particularly long-term prospective studies. Third, since most of the included studies excluded severe or critical patients with coronavirus diseases, our study may underestimate the severity of psychiatric symptoms of patients with COVID19 and SARS. Fourth, the present study focused on psychiatric symptoms based only on a self-rating scale only, the SCL-90-R, so data on psychiatric symptoms of COVID-19 patients who were illiterate and cognitively impaired were unavailable. Future studies using detailed comprehensive psychiatric interviews would provide a more comprehensive picture of the profile of psychiatric symptoms of COVID-19. Fifth, the participants in the studies included in this meta-analysis were all Chinese patients with coronavirus diseases. Because sociocultural 
factors play an important role in the clinical manifestations of mental health problems, caution is needed when generalizing our results to COVID-19 patients in countries other than China. Finally, as shown in Table 2, high levels of heterogeneity were detected in most metaanalyses. However, due to the limited number of included studies in each meta-analysis, we were not able to perform subgroup analysis to identify factors associated with the severity of each psychiatric symptom. It is worth noting that our study provided only an overall profile of the psychiatric symptoms of COVID-19, not a detailed profile of psychiatric symptoms.

In summary, a wide spectrum of severe psychiatric symptoms occur in COVID-19 patients, and most symptoms are still mild-to-medium during very early recovery. Based on SCL-90-R data from SARS patients and survivors, the severity of psychiatric symptoms of COVID-19 may decline following discharge, but some symptoms could persist for a long time during the convalescent stage. These findings suggest the urgent need of patients for extensive mental health services and psychological crisis intervention during the acute stage of COVID-19. Furthermore, it is also important to periodically monitor the psychiatric symptoms and provide psychosocial support, and psychiatric consultation and treatment (when necessary) for COVID-19 survivors during their convalescent stage. In addition, more research is needed to examine the longitudinal changes in psychiatric symptoms of COVID-19 survivors.

\section{Acknowledgements}

The study was supported by the National Natural Science Foundation of China (71774060, B.-L.Z., PI).

\section{Competing interests}

The authors declare no competing interests.

\section{Publisher's note}

Springer Nature remains neutral with regard to jurisdictional claims in published maps and institutional affiliations.

Received: 3 October 2020 Revised: 29 April 2021 Accepted: 5 May 2021 Published online: 17 May 2021

\section{References}

1. Varatharaj, A. et al. Neurological and neuropsychiatric complications of COVID19 in 153 patients: a UK-wide surveillance study. Lancet Psychiatry 7, 875-882 (2020).

2. Liguori, C. et al. Subjective neurological symptoms frequently occur in patients with SARS-CoV2 infection. Brain Behav. Immun. 88, 11-16 (2020).

3. Zarghami, A., Farjam, M., Fakhraei, B., Hashemzadeh, K. \& Yazdanpanah, M. H. A report of the telepsychiatric evaluation of SARS-CoV-2 patients. Telemed. J. E Health 26, 1461-1465 (2020).

4. Ma, Y. F. et al. Prevalence of depression and its association with quality of life in clinically stable patients with COVID-19. J. Affect Disord. 275, 145-148 (2020).

5. Xie, Q. et al. COVID-19 patients managed in psychiatric inpatient settings due to first-episode mental disorders in Wuhan, China: clinical characteristics, treatments, outcomes, and our experiences. Transl. Psychiatry 10, 337 (2020).
6. Turan, S. et al. Characteristics and outcomes of COVID-19 inpatients who underwent psychiatric consultations. Asian J. Psychiatr. 57, 102563 (2021).

7. Parra, A. et al. Psychotic symptoms in COVID-19 patients. A retrospective descriptive study. Psychiatry Res. 291, 113254 (2020).

8. Lu, S. et al. First report of manic-like symptoms in a COVID-19 patient with no previous history of a psychiatric disorder. J. Affect Disord. 277, 337-340 (2020).

9. $\mathrm{Hu}, \mathrm{Y}$. et al. Factors related to mental health of inpatients with COVID-19 in Wuhan, China. Brain Behav. Immun. 89, 587-593 (2020).

10. Nalleballe, K. et al. Spectrum of neuropsychiatric manifestations in COVID-19. Brain Behav. Immun. 88, 71-74 (2020).

11. Chen, S. P., Chang, W. P. \& Stuart, H. Self-reflection and screening mental health on Canadian campuses: validation of the mental health continuum model. BMC Psychol. 8, 76 (2020).

12. World Health Organization. Coronavirus disease (COVID-2019) situation reports https://www.who.int/emergencies/diseases/novel-coronavirus-2019/situationreports (2021).

13. Li, Z. et al. Rehabilitation needs of the first cohort of post-acute COVID-19 patients in Hubei, China. Eur. J. Phys. Rehabilitation Med. 56, 339-344 (2020).

14. Worldometer. COVID-19 coronavirus pandemic https:/www.worldometers. info/coronavirus/ (2021).

15. Garriques, E. et al. Post-discharge persistent symptoms and health-related quality of life after hospitalization for COVID-19. J. Infect. 81, e4-e6 (2020).

16. Huang, C. et al. 6-month consequences of COVID-19 in patients discharged from hospital: a cohort study. Lancet 397, 220-232 (2021).

17. Derogatis, L. R. SCL-90 : Administration, Scoring and Procedures Manual-l for the Revised Version and other Instruments of the Psychopathology Rating Scale Seriesed (Johns Hopkins University School of Medicine, Clinical Psychometrics Research Unit, 1983).

18. Zhong, B. L. et al. Prevalence of psychological symptoms in contemporary Chinese rural-to-urban migrant workers: an exploratory meta-analysis of observational studies using the SCL-90-R. Soc. Psychiatry Psychiatr. Epidemiol. 48, 1569-1581 (2013).

19. Pan, X. et al. Coronavirus disease 2019-related mental health: research progress. Acad. J. Second Mil. Med. Univ. 41, 303-306 (2020).

20. Hui, J., Zeng, P., Liang, H. \& Sun, J. Effects of psychological crisis intervention of Neuman health system model on anxiety and depression in patients with new type of coronavirus. J. Int. Psychiatry 47, 637-641 (2020).

21. Xing, H., Xia, Y., Chen, H., Wang, H. \& Zhu, J. Investigation on psychological status of patients with COVID-19 and influencing factors in Ningbo City. Hospital Manag. Forum 37, 115-117 (2020).

22. Qian, J. et al. Psychological crisis intervention on the psychological status of 2019-ncov patients. China J. Health Psychol. 29, 101-104 (2021).

23. Wang, M., Yang, J., Zhao, X., Ma, J. \& Li, J. Influence of empathy psychological intervention on coping style and mental health maintenance of patients with critical COVID-19. Lab. Med. Clin. 17, 3499-3501 (2020).

24. Gardner, P. \& Moallef, P. Psychological impact on SARS survivors: critical review of the english language literature. Can. Psychol. 56, 123-135 (2015).

25. Munn, Z., Moola, S., Lisy, K, Riitano, D. \& Tufanaru, C. Methodological guidance for systematic reviews of observational epidemiological studies reporting prevalence and cumulative incidence data. Int. J. Evid. Based Health. 13, 147-153 (2015).

26. Luo, W., Zhong, B. L. \& Chiu, H. F. Prevalence of depressive symptoms among Chinese university students amid the COVID-19 pandemic: a systematic review and meta-analysis. Epidemiol. Psychiatr. Sci. 30, e31 (2021).

27. Durlak, J. A. How to select, calculate, and interpret effect sizes. J. Pediatr. Psychol. 34, 917-928 (2009).

28. Tong, H. A research of twenty years' vicissitude: SCL-90 and its norm. Psychol. Sci. 33, 928-930 (2010).

29. Liu, Y. et al. A survey of mental symptoms of Chinese population based on SCL-90. Chin. Ment. Health J. 32, 437-441 (2018).

30. $H u, X ., M a, D ., L i u, G .$, Zhang, S. \& Wang, W. Nursing strategy for and an investigation of the mental health status of COVID-19 patients in shelter hospitals. J. Qilu Nurs. 26, 116-117 (2020).

31. Liu, X. \& Yu, S. Analysis of psychological characteristics of COVID-19 patients and nursing interventions. Med. J. Natl Defending Forces Southwest China $\mathbf{3 0}$ 496-499 (2020).

32. Mei, J. et al. Analysis of psychological and sleep state of medical stuff with novel coronavirus pneumonia. Her. Med. 39, 345-349 (2020).

33. Qin, X. et al. Mental health status of patients with coronavirus disease 2019 in Changsha. J. Cent. South Univ. (Med. Sci.) 45, 657-664 (2020). 
34. Wang, $H$. et al. Psychological stress status and its influencing factors among inpatients with COVID-19. J. Nurs. Sci. 35, 75-79 (2020).

35. Xi, C. Psychological investigation analysis and intervention of patients with pneumonia infected by COVID-19. Smart Healthc. 6, 93-95 (2020).

36. Yang, D. et al. Study on psychological status and psychological intervention of COVID-19 patients in Fangcang Shelter hospital. China J. Health Psychol. 29 560-564 (2021).

37. Gong, Z., Zhang, C., Xia, J., Xiao, C. \& Wang, H. Investigation on psychological status of medical staff infected by SARS-CoV-2. Chin. Nurs. Res. 34, 4307-4308 (2020).

38. Peng, $\mathrm{C}$. et al. Association between mental health status and social support in patients with severe acute respiratory syndrome. Chin. J. Clin. Rehabilitation $\mathbf{9}$, 60-61 (2005).

39. Sun, H. \& Ren, X. Psychological analysis for the medical staff suffering from severe acute respiratory syndrome. Chin. J. Clin. Rehabilitation 8, 7609-7611 (2004).

40. Wang, Z. et al. Psychological crisis intervention model in Xiaotangshan Hospital of PLA. Chin. Ment. Health J. 17, 587-590 (2003).

41. Wang, F. et al. Comparison of psychological status between patients with SARS and physicians. Nurses Treat. Sars. Chin. Ment. Health J. 17, 532-533 (2003).

42. Wang, J. et al. Psychological and physical presentations of severe acute respiratory syndrome. Chin. J. Intern. Med. 44, 30-33 (2005).

43. Xiao, R. et al. Investigation on psychological condition of patients with severe acute respiratory syndrome. Chin. J. Clin. Rehabilitation 8, 4166-4167 (2004).

44. Yang, H. Exploration of Response of Psychology and Psychological Nursing Intervention in SARS Patients (Shanxi Medical University, 2004).

45. Hu, H., Li, M., Zhou, L., Zhang, H. \& Wang, T. Effect of coping style and social support on the psychologic status in patients with severe acute respiratory syndrome. Chin. J. Clin. Rehabilitation 8, 1022-1023 (2004).
46. Liu, S., Gao, H. \& Hu, Y. Changes of the psychic stress state of patients with severe acute respiratory syndrome (SARS). Mod. Prev. Med. 34, 3447-3448, 3452 (2007).

47. Wang, W. et al. Analytical report on SCL-90 of 103 SARS patients. Chin. J. Behav. Sci. 12, 548-551 (2003).

48. Gao, H. et al. A path analysis of mentality for the SARS patients after discharge. Chin. J. Rehabilitation Med. 21, 832-834 (2006).

49. Kuang, $\mathrm{H}$. et al. Analysis of the results of symptom checklist 90 for convalescent patients with severe acute respiratory syndrome-a report of 62 cases. N. Med. 35, 737-738 (2004).

50. Lin, Z., Zuo, X. \& Zhong, S. Mental status of recovered SARS patients. Chin. Ment. Health J. 18, 129-131 (2004).

51. Moriguchi, T. et al. A first case of meningitis/encephalitis associated with SARSCoronavirus-2. Int. J. Infect. Dis. 94, 55-58 (2020).

52. Benameur, K. et al. Encephalopathy and encephalitis associated with cerebrospinal fluid cytokine alterations and coronavirus disease, Atlanta, Georgia, USA, 2020. Emerg. Infect. Dis. 26, 2016-2021 (2020).

53. De Felice, F. G., Tovar-Moll, F., Moll, J., Munoz, D. P. \& Ferreira, S. T. Severe acute respiratory syndrome coronavirus 2 (SARS-CoV-2) and the central nervous system. Trends Neurosci. 43, 355-357 (2020).

54. Zhang, C. L., Xu, Y. M. \& Zhong, B. L. The association between smoking and loneliness among Chinese university freshmen. Ann. Transl. Med. 8, 649 (2020).

55. Sharma, A. Chloroquine paradox may cause more damage than help fight COVID-19. Microbes Infect. 22, 154-156 (2020).

56. Janes, M., Kuster, S., Goldson, T. M. \& Forjuoh, S. N. Steroid-induced psychosis. Proc. (Bayl. Univ. Med Cent.) 32, 614-615 (2019).

57. Zhou, M. Y. et al. From SARS to COVID-19: what we have learned about children infected with COVID-19. Int. J. Infect. Dis. 96, 710-714 (2020).

58. Zhong, B. L. et al. Mental health problems, needs, and service use among people living within and outside Wuhan during the COVID-19 epidemic in China. Ann. Transl. Med. 8, 1392 (2020). 